The development of practical recommendations for shift work, the definition of duration and intensity of night shift rotation and shift schedules significance, taking into account the individual characteristics of workers (for example, the chronotype), requires further studies, as well as the issue of potential carcinogenic risk of shift work

\section{SHIFT WORK AND WORKPLACE VIOLENCE AGAINST HEALTHCARE WORKERS IN PSYCHIATRIC WARDS: A CASE-CONTROL STUDY}

G d'Ettorre ${ }^{1 *}, V$ Pellicani'. ' Local Health Authority of Brindisi (ASL BR), Department of Occupational Medicine, Italy

\subsection{6/oemed-2018-ICOHabstracts.1389}

${ }^{2}$ Local Health Authority of Lecce (ASL LE), Department of Mental Health, Italy

Introduction Workplace violence (WPV) towards healthcare workers (HCWs) employed in psychiatric wards (PWs) represeants a concern for healthcare organisations, globally. To date there is a lack of scientific data about the relationship between work-shifts and the occurrence of WPV against PW HCWs. The aim of the present study was to investigate the relationship between work shift schedules and WPV among registered nurses (RNs) working on non-traditional shifts, including nights and 12 hour shifts.

Methods The authors conducted a cross-sectional nested casecontrol analysis of data regarding the episodes of WPV perpetrated by patients or their relatives against RNs employed in two PWs, in the period between January-December 2016.

Results The one-year incidence of WPV was 31,50 per 100 Full Time Equivalent (FTE) positions. Cumulative nightshifts were significant for 3 or more nightshifts compared to working less than 3 nightshifts during the 7 days prior to the occurrence of WPV; additionally, RNs working 9 or more night-shifts showed higer risk of experiencing WPV compared to RNs working less than 4 night-shifts in the previous 28 days.

Discussion In the present study the occurrence of WPV against PW RNs was significantly correlated with shift- work; as consequence, the findings support the need of organisational interventions aimed at preventing the WPV and targeted on the management of shift-work schedules, with the aim of:

- limiting the night shifts up to two per week and up eight per month;

- adopting constant forward-rotating shift schedules.

\section{ASSOCIATION BETWEEN SOCIAL JETLAG AND OBESITY AMONG MALE FACTORY WORKERS IN JAPAN}

Reiko Kuroda*, Kenya Yamamoto, Yasushi Okubo. Division for Environment, Health and Safety, the University of Tokyo, Tokyo, Japan

\subsection{6/oemed-2018-ICOHabstracts. 1390}

Introduction Obesity is a major risk factors for noncommunicable diseases such as cardiovascular diseases, musculoskeletal disorders, and some cancers, and this is preventable. Not only sleep duration or chronotype have been associated with body mass index (BMI), but also circadian misalignment 'social jetlag' may be possibly associated in recent studies. We investigated whether social jetlag were associated with obesity, independent from sleep duration or chronotype.

Methods A cross-sectional study was conducted among 1357 male workers who works at an electrical manufacturer from April 2014 to January 2015. Body height and weight, smoking status and frequency of alcohol drinking were collected via annual medical check-up.

Sleep duration, chronotype, and other work- or life- related factor were collected through an additional questionnaire. Social jetlag (SJ) was calculated as the absolute difference between mid-sleep time on weekdays and weekends and were categorised into four groups. Logistic regression analysis was performed to estimate the odds ratio (OR) for 1) obesity $\left(B M I \geq 30 \mathrm{~kg} / \mathrm{m}^{2}\right.$ ) and 2) abdominal obesity (Waist circumference $\geq 90 \mathrm{~cm}$ ) for each SJ category (SJ $<1$ hour as reference) with adjustments for demographic, work- and life- related factors. A P value $<0.05$ was considered significant. Data were analysed using SPSS Ver.22.0.

Results Extreme SJ category ( $\leq 3$ hours) had significant high OR (95\% confidence interval [CI]) of 1) obesity and 2) central obesity after controlling for age, presence of night shift work, chronotype, sleep duration. OR (95\% CI) were 3.68 (1.49-9.11), and $2.48(1.28-4.79)$, respectively. We found no impact of SJ on BMI or abdominal obesity after controlling for additional other variables.

Conclusion It was found that extreme social jetlag possibly associated with high OR of obesity or abdominal obesity, independent from sleep duration and chronotype. However, there were no associations between social jetlag and BMI or abdominal obesity, when we considered other life-style variables.

\section{THE IMPACT OF SHIFTWORK ON SLEEP QUALITY AMONG NHS NURSES}

${ }^{1} \mathrm{~K}$ McDowall ${ }^{*}{ }^{1} \mathrm{E}$ Murphy, ${ }^{2} \mathrm{~K}$ Anderson. ${ }^{1}$ Occupational Health Service, Newcastle Hospitals NHS Foundation Trust, UK; ${ }^{2}$ Neurology Department, Newcastle Hospitals NHS Foundation Trust, UK

\subsection{6/oemed-2018-ICOHabstracts. 1391}

Introduction Shiftwork is common amongst nurses and it is known to be a workplace hazard as it may cause poor sleep quality, which can impact adversely on the health and safety of nurses and their patients. The aims of this study were to identify and describe the association between poor sleep quality and shiftwork in nurses. Additionally, to explore factors that contribute to poor sleep quality and to assess the awareness of support from Occupational Health $(\mathrm{OH})$.

Methods Cross-sectional study of nurses at a National Health Service Foundation Trust, February to March 2016. Data was collected via an online questionnaire. Sleep quality was measured using the Pittsburgh Sleep Quality Index.

Result 888 nurses participated; 34\% response rate. The prevalence of poor sleep quality was $78 \%$ (95\% CI: 0.748 to 0.813 ) in the shift working nurses (SWNs), compared to $59 \%$ (95\% CI: 0.503 to 0.678 ) in the non-shift working nurses (NSWNs). There was a mean sleep quality score difference of 1.58 between the SWNs and the NSWNs, which was statistically significant, $\mathrm{p}<0.001$ (95\% CI: 0.913 to 2.246). Undertaking shiftwork was the only significant association with poor sleep quality, when controlling for the other variables of age, gender and number of years worked, OR $0.410 \quad p<0.001$ (95\% CI: 0.265 to 0.634 ). 
Discussion There is a higher prevalence of poor sleep quality in SWNs compared to NSWNs. OH should be aware of any form of shiftwork as an important risk factor for poor sleep, as well as Trust managers acknowledging this workplace hazard. $\mathrm{OH}$ can provide staff with support for good sleep practice which aims to lead to healthier nurses.

\section{STRATEGIES OF TIME MANAGEMENT IN THE DAILY ROUTINE OF MEDICAL RESIDENTS OF INTERNAL MEDICINE IN A PUBLIC UNIVERSITY HOSPITAL}

Rafael Augusto Tamasauskas Torres*, Frida Marina Fischer. Department of Environmental Health, School of Public Health, University of São Paulo

\subsection{6/oemed-2018-ICOHabstracts. 1392}

Introduction Becoming a doctor is a complex process that usually consolidates during medical residency. This phase includes full immersion in professional activities with long working and studying hours. Junior doctors take care of patients in complex and difficult-to-manage care situations, both for their severity and institutional shortcomings. Although medical residency is recognised as an efficient form of professional training, there are several criticisms about work overload and long working hours.

Objective To identify strategies of time management in the daily routine of medical residents of Internal Medicine in a university hospital in São Paulo, Brazil.

Method In-depth interviews were conducted with eight randomly selected residents about their daily lives during the first year of residence.

Results The mean age was $25 \mathrm{y}$, being 4 men. Average hours of residence are $60 \mathrm{~h} /$ week. This excludes night shifts and work performed during days off. Over 8 months there is a 12 hour weekly night shift; and during 2 months there are 2 to 3 shifts per week. The reported highlights were: little time to accomplish domestic activities and commuting time to and from the hospital/home. Reduced time for leisure, sleep and study activities. Six out of eight participants reported activities during night shifts for supplementation of income. Some strategies for managing time were: moving to places close to the hospital, hiring cleaning ladies, eating out at the hospital restaurant, keeping a social circle restricted to other residents. The need for income supplementation was reported as a time management constraint.

Discussion Time management strategies during residence include measures to save time such as hiring third persons to perform domestic activities, and living close to the hospital. Some of the reported consequences are: the reduction of time to study, the restriction in the social relations and working odd-hours/days to supplement their income.

\section{URINARY 8-HYDROXYDEOXYGUANOSINE LEVELS OF EMERGENCY MEDICAL SHIFT WORKERS ARE HIGHER THAN THOSE OF FACTORY WORKERS}

Shintaro Watanabe*, Kazuaki Kawai, Yunshan Li. Department of Environmental Oncology Institute of Industrial Ecological Sciences, University of Occupational and Environmental Health, Japan

10.1136/oemed-2018-ICOHabstracts. 1393
Introduction Shiftwork is known as a risk factor of breast cancer and prostate cancer. In fact, the International Agency for Research on Cancer (IARC) classified shiftwork as Class 2A. One mechanism is that shiftwork increases cellular oxidative stress and results in DNA damage. Urinary 8-hydroxydeoxyguanosine $(8-\mathrm{OHdG})$ is frequently analysed as an oxidative stress marker. However, there have been few studies investigating the association of shiftwork with urinary 8-OHdG levels. In this study, we investigated the association between shiftwork with oxidative stress and urinary 8-OHdG levels.

Methods Urine samples from eight medical shift workers were collected before and after the night shift. The 8-OHdG levels were measured with a high-performance liquid chromatography (HPLC) system equipped with an electrochemical detector.

Results The mean level of urinary $8-\mathrm{OHdG}$ ( $\mu \mathrm{g} / \mathrm{ng}$ creatinine) in this study was $5.40 \pm 1.70$, and was higher than that of factory workers in a past study. There were no significant differences in the urinary $8-\mathrm{OHdG}$ levels before and after the night shift work.

Conclusion These results suggest that shiftwork, strain, and chronic fatigue lead to oxidative stress and DNA damage. The urinary $8-\mathrm{OHdG}$ level may be a useful biomarker, as an evaluating factor for chronic fatigue in regard to shiftwork.

\section{ASSOCIATION OF SHIFT-WORK, DAYTIME NAPPING, AND NIGHTTIME SLEEP WITH CANCER INCIDENCE AND CANCER-CAUSED MORTALITY IN DONGFENG-TONGJI COHORT STUDY}

${ }^{1}$ Yansen Bai ${ }^{*}$, 'Xiaoliang Li, 'Ke Wang, 'Shi Chen, 'Suhan Wang, 'Zhuowang Chen, ${ }^{1}$ Xiulong Wu, 'Wenshan Fu, 'Sheng Wei, 'Jing Yuan, 'Ping Yao, 'Xiaoping Miao, ${ }^{1}$ Xiaomin Zhang, ${ }^{1}$ Meian $\mathrm{He},{ }^{2}$ Handong Yang, ${ }^{1}$ Tangchun Wu, ${ }^{1}$ Huan Guo. ${ }^{1}$ Department of Occupational and Environmental Health, Tongji Medical College, Wuhan, China; ${ }^{2}$ Dongfeng Motor Corporation and Hubei University of Medicine, Shiyan, China

\subsection{6/oemed-2018-ICOHabstracts.1394}

Introduction More than 30\% of cancer deaths could be prevented by modifying or avoiding risk factors, such as unhealthy living habits, including night-shift work, daytime napping, and nighttime sleep. We aimed to investigate the independent and combined effects of these three sleep habits on cancer incidence and cancer-caused mortality among middle-aged and older Chinese in the Dongfeng-Tongji Cohort Study (27 009 retired workers were recruited from Dong-Feng Motor Corporation (DMC) in 2008, and 25978 participants were successfully followed-up during the first follow-up period from June, 2013 to October, 2013). During 114162 person-years of follow up, we identified a total of 1251 cancer cases and 379 cancer-caused deaths.

Methods Information on sleep habits, cancer incidences and mortalities were collected at baseline 2008. Self-reported cancer incidences and cancer-caused deaths were confirmed from DMC's health-care service system, which consists of five DMC-owned hospitals that covers all retired employees. Cox proportional hazards models were used to calculate the adjusted hazard ratios and 95\% confidence intervals (HRs, 95\% CIs).

Results Males experienced $\geq 20$ years of night-shift work, or no daytime napping had increased cancer incidence, when compared to males who did not have night-shift work or had daytime napping for $1 \sim 30 \mathrm{~min} \quad[\mathrm{HR}(95 \% \mathrm{CI})=1.27(1.01-$ 